\title{
errata
}

\section{Capture of an early fusion-active conformation of HIV-1 gp41}

Rika A. Furuta, Carl T. Wild, Yongkai Weng and Carol D. Weiss Nature Struct. Biol. 5, 276-279 (1998).

Several typographical errors were introduced into the 178HA peptide sequence during printing. The sequence is the same as the DP178 peptide, with the addition of a GGG linker and the HA epitope at the C-terminus. The correct sequence for this peptide is: YTSLIHSLIEESQNQQEKNEQELLELDKWASLWNWFGGGYPYDVPDYAGPG. We regret any confusion that this may have caused.

\section{Structure and switching of bacterial flagellar filaments studied by X-ray fiber diffraction}

Ichiro Yamashita, Kazuya Hasegawa, Hirofumi Suzuki, Ferenc Vonderviszt, Yuko Mimori-Kiyosue and Keiichi Namba Nature Struct. Biol. 5, 125-132 (1998).

The name of the first author was misprinted in the author list. It is spelled correctly above.

\section{correction}

\section{The alpha and beta of turning on a molecular switch}

Emil F. Pai Nature Struct. Biol. 5, 259-263 (1998).

Emil Pai would like to note that the structure of Sec7 was obtained in the laboratory of Jaqueline Cherfils, not in Pierre Chardin's laboratory as written. He apologizes for the error. 